Chiari-1 malformation. On examination, her neurocutaneous markers suggested classical KTS-presence of distinct, linear bordered port-wine stains. She had lymphoedema of bilateral lower limbs and disproportionately enlarged feet with club feet deformity. Solid organ involvement and congenital heart disease were ruled out. Adequate blood products were arranged in view of anticipated blood loss. Standard anesthetic induction was performed. In view of Chiari malformation and anticipated soft tissue changes in the airway, intubation was performed using C-MAC videolaryngoscope. Patient was positioned carefully and surgery was performed uneventfully.

Conclusion: Patients with KTS pose challenges during vascular access necessitating use of ultrasonography to diagnose and avoid inadvertent injury to superficial arteriovenous fistulous malformations. Similar concerns exist during scalp block regarding inadvertent local anesthetic injection into occult subcutaneous scalp hemangiomas. Presence of associated Chiari-1 malformation and proneness for pathological fractures requires care during intubation and positioning. Oropharyngeal neurofibromatosis lesions warrant preoperative airway examination using indirect laryngoscopy to understand the potential airway difficulty and formulate intubation plan. Calvarial hyperostosis can result in difficulty during craniotomy. Proximity of the hemangioma to the superior sagittal sinus places the patient at risk of venous air embolism.

\section{A036 Role of NIRS in a Child with Moyamoya Disease for Encephaloduroarteriomyosynangiosis (EDAMS): A Case Report}

Samuel Chandran, ${ }^{1}$ Karen R. Lionel, ${ }^{1}$ Ramamani Mariappan ${ }^{1}$ ${ }^{1}$ Department of Anaesthesia, Christian Medical College (CMC), Vellore, Tamil Nadu, India

Background: Moyamoya, "puff of smoke" disease (MMD), is a rare cerebrovascular disease with progressive stenosis of intracranial blood vessels. Anesthetic goal of revascularization surgery is to maintain the cerebral oxygen supply and demand. We wanted to report a case of NIRSbased anesthetic management of encephaloduroarteriomyosynangiosis (EDAMS).

Case Description: An 8-year-old boy weighing $24 \mathrm{~kg}$ presented with two episodes of transient ischemic attacks (TIAs), diagnosed with MMD, planned for EDAMS. Overnight intravenous fluid (IVF) was given to prevent dehydration and diazepam premedication was given for anxiolysis. In the operating room, with ECG, EtCO2, SPO2, NIRS, and NIBP monitoring were attached; induction and intubation were performed using propofol, fentanyl, and atracurium. Postintubation, arterial BP, temperature, and urine output monitoring was established. Regional scalp block with $0.2 \%$ ropivacaine was given for analgesia. Anesthesia was maintained using air, oxygen, and sevoflurane. Depth of anesthesia, brain oxygenation, and hemoglobin trends were monitored using the patient state index, NIRS, and noninvasive hemoglobin analyzer using SedLine, Masimo. MAP, CO2, temperature, and depth of anesthesia were tightly controlled to keep the cerebral oxygenation close to the baseline. Repeat regional scalp block, fentanyl infusion was given for postoperative analgesia. He was discharged on the POD 4 without neurological deficit.

Conclusion: MMD has limited cerebrovascular reserve with propensity to develop cerebral ischemia during the perioperative period. In our case, the balance between the cerebral oxygen supply and demand was strictly maintained by monitoring the cerebral oxygenation using NIRS. Various factors, such as MAP, EtCO2, temperature, depth of anesthesia, and intraoperative hematocrit were controlled within normal limit to maintain the cerebral oxygenation close to baseline throughout surgery which aided for the successful outcome.

\section{A037 Utility of Compass 31 Questionnaire to Predict the Autonomic Dysfunction and Intraoperative Hemodynamic Fluctuations and Correlate it with CASS Score in Patients with Compressive Cervical Myelopathy Tanushree, ${ }^{1}$ Rosen R. Mathew, ${ }^{1}$ Ramamani Mariappan ${ }^{1}$ ${ }^{1}$ Department of Anaesthesia, Christian Medical College (CMC), Vellore, Tamil Nadu, India}

Background: To assess utility of COMPASS-31 questionnaire as screening tool for diagnosing autonomic dysfunction (AND) and correlate it with modified CASS score to predict the severity of AND, intraoperative hemodynamic fluctuations in patients undergoing surgery for compressive cervical myelopathy (CCM).

Materials and Methods: After obtaining IRB approval and informed consent, 42 ASA I-II patients, aged 18 to 70 years, who underwent decompression for CCM were recruited. In the preoperative period, COMPASS 31 questionnaire was given in the language they could comprehend, and the total score was calculated. They underwent autonomic function tests and modified CASS score was calculated. During intraoperative period, standard anesthesia protocol followed and hemodynamics were noted at regular intervals throughout the surgery. Ephedrine/phenylephrine boluses or noradrenaline infusion was given to maintain a target BP within 20\% of baseline. Amount of vasopressor used was recorded.

Results: The mean age of the patients studied was $48.76 \pm 10.69$ years. Fifty percent of patients had Nurick's grades 2 and 3, and the rest had Nurick's grades 4 and 5. All patients had AND varying from mild (46\%), moderate (12\%) to severe (42.9\%). The median COMPASS-31 score (IQR: 25-75\%) was 19 (6-33). Receiver operating characteristic (ROC) analysis revealed COMPASS-31 had fair accuracy with area under the curve $($ AUC $)=0.738(p=0.009)$. Total COMPASS-31 score of 30 had sensitivity of $52.2 \%$ and specificity of $89.5 \%$ to detect moderate to severe AND. The median (IQR: $25-75 \%)$ Modified CASS score was 3 (2-5). Moderately positive correlation $0.383(p=0.05)$ found between CASS and COMPASS-31 score. Nurick's grade showed positive correlation with CASS/COMPASS-31with correlation coefficient of 0.35 ( $p=0.023), 0.48(p=0.001)$, respectively. In patients with severe AND had significant hemodynamic fluctuation compared with mild/moderate AND. 